\section{bendamustin}

\section{Multizentrische Studien bestätigen hohe Effektivität von Bendamustin (Ribomustin $^{\circledR}$ ) bei Lymphomen}

Auf dem ASH-Kongress in San Diego wurden im Dezember 2004 die Ergebnisse zweier multizentrischer Studien mit Bendamustin bei indolenten NHL präsentiert. Bendamustin wurde mit Mitoxantron und dem CD20-Antikörper Rituximab (BMRSchema) in einer Pilotstudie von PD Dr. R. Weide, Koblenz, erstmals in dieser Kombination systematisch eingesetzt. Aufgrund der Ergebnisse bei rezidivierten oder refraktären Patienten mit indolenten NHL untersuchte die Deutsche Studiengruppe indolente Lymphome (GLSG) dieses BMR-Schema multizentrisch in einer Rezidivstudie. Belegt wird die hohe Effek- tivität des BMR-Schemas bei 41 auswertbaren Patienten mit fortgeschrittenen rezidivierten/refraktären indolenten NHL: Gesamtansprechrate 93\%, CR-Rate 40\%. Die Therapie wurde insgesamt gut vertragen, die Toxizität beschränkte sich auf hämatologische Nebenwirkungen (Grad 3 oder 4: 78\%). 3 Patienten wurden wegen febriler Neutropien bzw. eines Infekts hospitalisiert. 16 der 41 Patienten waren mit Rituximab vortherapiert; von diesen sprachen $14(88 \%)$ auf das BMR-Schema an, davon $9(56 \%)$ mit einer CR.

Die ersten Daten einer zulassungsrelevanten Studie in den USA wurden von Friedberg et al. präsentiert: Bendamustin (SDX-105; Salmedix, USA) wurde in einer multizentrischen Phase-II-Studie als Monotherapie bei Rituximab-refraktären Patienten mit indolenten oder sekundär hochmalignen NHL eingesetzt. 32 auswertbare Patienten hatten im Median 3,5 Vortherapien bekommen: $120 \mathrm{mg} / \mathrm{m}^{2}$ Bendamustin, Tag $1+2$, alle 3 Wochen, $5 \mathrm{Zy}-$ klen. Gesamtansprechrate: 75\%, CR-
Rate: $25 \%$. Grad-3- oder -4-Toxizitäten: 44\% Neutropenie, 8\% Anämie, 19\% Thrombopenie. Weitere Zulassungsstudien in den USA sind geplant.

Neuere Analysen zum Wirkmechanismus bestätigen, dass Bendamustin ein anderes Aktivititätsprofil zeigt als andere alkylierende Substanzen. Auch wird ein für diese Substanzen bisher unbekannter zytotoxischer Effekt von Bendamustin beschrieben: Die «mitotische Katastrophe», ein Mechanismus, der eher mit Vinca-Alkalioden oder Taxanen in Verbindung gebracht wird. Diese Daten deuten darauf, dass Bendamustin zusätzliche zytotoxische Aktivität entfalten kann, wenn der klassische Apoptoseweg gehemmt ist, und können erklären, warum Bendamustin bei (Alkylanzien-)refraktären Patienten erfolgreich eingesetzt werden kann.

Weitere Informationen bei: ribosepharm GmbH, Dr. Almut-J. Wegner Tel. +49 8945 450-201, Fax -266 E-mail almut.wegner@ribosepharm.de

\section{Überlegene Wirksamkeit von Alemtuzumab bei Hochrisiko-CLL}

Im Rahmen der Jahrestagung der American Society of Hematology (ASH) 2004 wurden aktuelle Ergebnisse der CLL2HStudie der Deutschen CLL-Studiengruppe präsentiert.

Eine besondere therapeutische Herausforderung stellen CLL-Patienten dar, bei denen das Tumorsuppressorgen p53 durch eine Deletion im Chromosom 17 (17p-Deletion) ausgeschaltet wurde. Patienten mit diesem Hochrisikofaktor haben eine sehr ungünstige Prognose und sprechen meist nicht auf konventionelle Chemotherapien oder den Anti-CD20-Antikörper Rituximab an. Im Gegensatz hierzu erwies sich der AntiCD52-Antikörper Alemtuzumab (MabCampath $^{\circledR}$ ) auch bei dieser Hochrisikogruppe als überaus wirksam. Bisher wurden 50 stark vorbehandelte refraktäre CLL-Patienten, die alle auf das Standardmedikament Fludarabin $\left(\right.$ Fludara $^{\circledR}$ ) nicht oder kürzer als 6 Monate angesprochen hatten, mit $3 \times 30 \mathrm{mg}$ Alemtuzumab wöchentlich für bis zu 12 Wochen behandelt. Statt einer mehrstündigen Infusion wurde der Antikörper subkutan appliziert, was für die Patienten deutlich angenehmer war. Fast ein Drittel des Studienkollektivs (29\%) wies den Hochrisikofaktor 17p-Deletion auf, und über die Hälfte dieser $\mathrm{Pa}$ tienten sprach gut auf die AlemtuzumabBehandlung an. Damit übertraf die Ansprechrate dieser Hochrisikogruppe sogar die des Gesamtkollektivs, die bei 37\% lag. Die progressionsfreie Zeit von 10,8 Monaten und ein medianes Gesamtüberleben von 17,4 Monaten bestätigten die hohe Wirksamkeit der Alemtuzumab-Therapie. Weitere Informationen bei:

Medac Schering Onkologie GmbH

Tel. +49 8974514430

E-mail k.grassmann@mso-pharma.de

\title{
PharmaTicker+++ PharmaTicker+++ PharmaTicker+++ PharmaTicker+++
}

Merck Pharma GmbH. In den Leitlinien der Deutschen Gesellschaft für Verdauungs- und Stoffwechselkrankheiten (DGVS) wird der EGFR-Antikörper Erbitux ${ }^{\circledR}$ bereits ein halbes Jahr nach seiner Zulassung für die Behandlung von fortgeschrittenem Darmkrebs empfohlen. Mit dem Empfehlungsgrad A und der Evidenzstärke 1b sowie starkem Konsens entspricht diese Empfehlung dem Empfehlungsgrad der bereits etablierten Darmkrebstherapien. Weiter Informationen bei:

Merck Pharma GmbH, Dr. Roland Zagler

Tel. +49 $615172-2545$

E-mail pressestelle@merck-pharma.de
Gilead Sciences GmbH. Beim 55. Kongress der American Association for the Study of Liver Diseases (AASLD) in Boston wurde eine Langzeitstudie (Studie 437) mit dem antiviral wirksamen Adefovirdipivoxil (Hepsera ${ }^{\circledR}$ ) bei Patienten mit chronischer Hepatitis B präsentiert: Innerhalb von 3 Jahren kommt es bei $>43 \%$ der Patienten, die mit wt-Virus infiziert waren, zu einer HBeAg-Serokonversion. Die Substanz war auch in der Langzeittherapie gut verträglich. Gilead Sciences GmbH, Dr. D. Langenbacher-Nießing Tel. +49 $89899890-54$

E-mail dlangenbacher@gilead.com
Die Pfizer Pharma GmbH hat bei der europäischen Behörde EMEA die Zulassung für Aromasin $^{\circledR}$ (Exemestan) zur adjuvanten Therapie des Brustkrebs mit positivem oder unbekanntem Hormonrezeptor-Status bei postmenopausalen Frauen, die $\geq 2$ Jahre Tamoxifen erhalten haben, beantragt. Bisher ist der steroidale AntiAromatase-Wirkstoff zur Therapie des fortgeschrittenen Mammakarzinoms nach Progress unter Anti-Östrogentherapie zugelassen. Pfizer Pharma GmbH, Dr. Thomas Menschik

Tel. +49 721 6101-9831

E-mail thomas.menschik@pfizer.com

\begin{tabular}{ll}
\hline KARGER & ( 2005 S. Karger GmbH, Freiburg \\
Fax +497614520714 & $\begin{array}{l}\text { Accessible online at: } \\
\text { www.karger.com/onk }\end{array}$
\end{tabular}




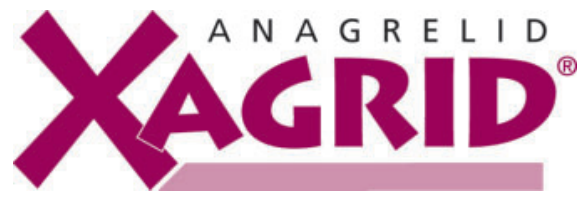

\section{Xagrid $^{\circledR}$ - zugelassen für die essentielle Thrombozythämie in Deutschland}

In Deutschland sind ca. 9000 Patienten von der essentiellen Thrombozythämie betroffen, einer Erkrankung des Knochenmarks, die meistens nach dem 50. Lebensjahr auftritt bzw. erkannt wird. Bei der Erkrankung, die zur Gruppe der chronisch myeloproliferativen Erkrankungen zählt, sind die Megakaryozyten stark vermehrt und deutlich vergrößert. In der Folge ist die Thrombozytenzahl stark erhöht (über 500.000 Thrombozyten pro $\mu \mathrm{l}$ ) und es besteht eine erhöhte Neigung zu arteriellen Thrombosen, die mit einem gesteigerten Risiko für Schlaganfall, Herzinfarkt und Verschluss der Arterien der Extremitäten einhergeht. Bei den Symptomen sind die am häufigsten auftretenden Beschwerden Mikrozirkulationsstörungen, Schwindel, Kopfschmerzen und Sehstörungen.

Bisher erfolgte die Therapie der essentiellen Thrombozythämie überwiegend mit Hydroxyurea und off-Label mit Interferon $\alpha$ oder auf der Basis des $\$ 73.3$ AMG mit Anagrelid (bisherige Bezeichnung Agrylin $\left.{ }^{\circledR}\right)$. Mit Hydroxyurea wird die Reduktion der Blutplättchen über die Hemmung der Ribonukleotidreduktase und der DNA-Synthese erreicht. Es handelt sich um eine zytotoxische, nicht-selektive Therapie. Der Wirkstoff hat jedoch eine leukämogene Potenz, die sich bei Monotherapie in einem Zeitrahmen von 4 bis 10 Jahren nach Behandlungsbeginn manifestieren kann. Interferon $\alpha$ wird - im Gegensatz zu Anagrelid und Hydroxyurea - parenteral appliziert und wirkt myelosuppressiv und immunmodulatorisch. Fieber und Fröstelgefühle sowie Erschöpfung sind die anhaltendsten und häufigsten Syndrome.

Xagrid $^{\circledR}$ (Wirkstoff Anagrelid) von Shire Deutschland hat jetzt von der EMEA als erstes anagrelidhaltiges Therapeutikum die europäische Zulassung für die Therapie der essentiellen Thrombozythämie erhalten. In den USA hat die FDA die Zulassung für Anagrelid in der Therapie essentielle Thrombozythämie bereits vor 7 Jahren erteilt. Seit dem Jahr 2000 hat Anagrelid den Orphan-Drug-Status.

Anagrelid wird seit über 10 Jahren in einem breiten Spektrum von Patienten erfolgreich in der Behandlung der essentiellen Thrombozythämie eingesetzt. Es wirkt in der postmitotischen Phase der Megakaryozyten-Entwicklung und reduziert deren Ploidie, Reife und Größe. Dadurch wird die Thrombozytenzahl verringert, ohne dass die anderen Zelllinien der Hämatopoiese unter einer chronischen Einnahme beeinflusst werden (Anagrelide Study Group, 1992). Pescatore und Lindley [2000] bestätigten diese Ergebnisse in ihren Untersuchungen. Die meisten Nebenwirkungen [Kopfschmerzen (14\%), Palpitationen (9\%), Flüssigkeitsretention (6\%), Übelkeit (6\%) und Diarrhö (5\%)] unter der Gabe von Xagrid ${ }^{\circledR}$ (Anagrelid) sind stoffklassenspezifisch und nehmen bei chronischer Einnahme ab.

Für die Behandlung der essentiellen Thrombozythämie mit Anagrelid sprechen vor allem die nachgewiesene kontrollierte
Plättchenreduktion, die Wirkspezifität sowie die nicht mutagene und zytotoxische Wirkung und daraus folgend kein leukämogenes Risikopotenzial.

Xagrid $^{\circledR}$ steht seit Januar für die Behandlung der essentiellen Thrombozythämie in Flaschen zu je 100 Hartkapseln (0,5 mg Anagrelid) zur Verfügung.

Weitere Informationen bei:

Shire Deutschland GmbH

Helge Scheider

Tel.: +49 2218804735

E-Mail hscheider@de.shire.com

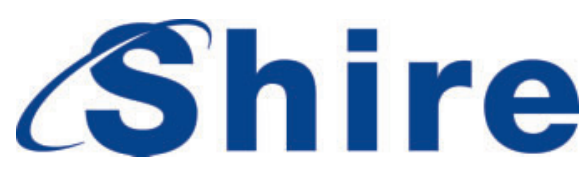

\section{PharmaTicker+++ PharmaTicker+++ PharmaTicker+++ PharmaTicker+++}

Janssen-Cilag GmbH. Mit dem neuen Durogesic ${ }^{\circledR}$ SMAT $12 \mu \mathrm{g} / \mathrm{h}$ steht der bewährte Wirksotff Fentanyl zur transdermalen Behandlung auch mäßig starker chronischer opioidpflichtiger Schmerzen zur Verfügung. In der Dosierung $12,5 \mu \mathrm{g} / \mathrm{h}$ bietet das transdermale Fentanyl $\mathrm{Pa}$ tienten mit tumor- und insbesondere nicht-tumorbedingten chronischen Schmerzen auch mittlerer Stärke anhaltende Wirksamkeit über 3 Tage bei guter Verträglichkeit.

Weitere Informationen bei:

Jannsen-Cilag GmbH, Frau Dr. Nicole Kubitz

Tel. +49 2137 955-0

E-mail nkubitz@jacde.jnj.com
Novartis Pharma GmbH. Auf dem Jahreskongress der ASH wurde im Dezember 2004 eine Aktualisierung der IRIS (International Randomized Interferon versus STI571)-Studie vorgestellt. Diese zeigt einen Zusammenhang zwischen einem raschen, frühen zytogenetischen Ansprechen und der Langzeitprognose während der Behandlung mit Glivec bei neu diagnostizierten Patienten in der chronischen Phase einer Ph+ CML.

Novartis Pharma GmbH, Dr. Michaela Paudler-Debus Tel. +49 911 273-12462

E-mail michaela.paudler-

debsus@pharma.novartis.com
Die sanofi-aventis Gruppe gab die Einführung von ABREAST (A Breast Cancer Registry of Adjuvant STrategies) bekannt. Dabei handelt es sich um das erste internationale prospektive System zur Registrierung von Patienten mit Brustkrebs im Frühstadium. Mit ABREAST wird man sich ein aktuelles Bild von der realen klinischen Praxis sowie von Patienten- und Krankheitsmerkmalen machen können.

Weitere Informationen bei:

Aventis Pharma Deutschland GmbH, Judith Kramer

Tel. +49 69 305-84412

E-mail judith.kramer@aventis.com 